\title{
A hiperhomocisteinemia como fator de risco cardiovascular: perspectivas atuais
}

\author{
The hyperhomocysteinemia as a cardiovascular \\ risk factor: actual perspectives
}

\author{
Sérgio Luiz Brasileiro Lopes ${ }^{1}$, Heloísa Helena Maia \\ Christovam Lopes ${ }^{2}$, Hélio Vannucchi ${ }^{3}$
}

Lopes SLB, Lopes HHMC, Vannucchi H. A hiperhomocisteinemia como fator de risco cardiovascular: perspectivas atuais. Rev Med (São Paulo). 2010 jan.-mar.;89(1):1-11.

RESUMO: A homocisteína, uma intermediária do metabolismo da metionina, é considerada, de forma ainda não consensual, como um fator de risco independente para cardiopatia isquêmica. Objetivando revisar a literatura, realizamos uma pesquisa bibliográfica acerca da associação entre a hiperhomocisteinemia e o risco cardiovascular. Conclusivamente, a hiperhomocisteinemia determina uma maior homocisteinilação protéica e molecular de LDL, induzindo o surgimento de auto-anticorpos específicos anti-proteína homocisteinilada, e o aumento dos níveis circulantes de LDL oxidada, com consequente incremento da propensão à ateromatose vascular. Essa associação parece depender, intrinsecamente, dos níveis séricos de dois importantes co-fatores envolvidos diretamente em seu metabolismo, o ácido fólico e a cobalamina. Indivíduos portadores de hiperhomocisteinemia têm reduzidos o risco cardiovascular e a mortalidade global se suplementados com esses co-fatores. Entretanto, essa ação benéfica parece estar restrita aos indivíduos que utilizam essas vitaminas como forma de prevenção primária, uma vez que, nos portadores de coronariopatia aterosclerótica sua utilização não conseguiu, na maioria dos estudos, minimizar a incidência de eventos isquêmicos ou a taxa de óbitos. Conclui-se que a hiperhomocisteinemia é um fator de risco independente para doença cardiovascular aterosclerótica, devendo, por isso, ser tratada precocemente, antes do desenvolvimento de sintomas isquêmicos, com a suplementação de ácido fólico e de cobalamina, objetivando o controle de seus níveis séricos e, consequentemente, do risco cardiovascular intrínseco. Esse controle clínico faz-se essencial principalmente naqueles indivíduos que, além da hiperhomocisteinemia, são portadores de outros fatores de risco clássicos para vasculopatia aterosclerótica.

DESCRITORES: Homocisteína; Hiper-homocisteinemia; Aterosclerose; Doenças Cardiovasculares; Fatores de risco.

1. Professor Adjunto do Departamento de Medicina do Centro de Ciências Biológicas e da Saúde da Universidade Federal de São Carlos, São Carlos, SP.

2. Coordenadora da Divisão de Cardiologia da Clínica Climatérium - Ribeirão Preto, SP.

3. Professor Titular do Departamento de Clínica Médica da Faculdade de Medicina de Ribeirão Preto da Universidade de São Paulo, Ribeirão Preto, SP.

Endereço para correspondência: Prof. Dr. Sérgio Luiz Brasileiro Lopes. Universidade Federal de São Carlos, Departamento de Medicina. Avenida Washington Luis, 235. CEP: 13565-905 - São Carlos - SP. e-mail: slblopes@ ufscar.br 


\section{INTRODUÇÃO}

A pesar de ser um tópico de discussão relativamente recente, novas evidências têm relacionado a hiperhomocisteinemia ao incremento do risco cardiovascular. Entretanto, ainda hoje, pouco se conhece acerca da fisiopatogenia dessa associação, existindo, predominantemente, duas correntes de opinião: a que imputa à hiperhomocisteinemia papel central nos mecanismos de aterogênese, e a que a considera apenas mais um marcador da presença de lesões vasculares ${ }^{1-5}$. Ainda hoje, essas dúvidas estão longe de serem sanadas, ainda que a maior parte da comunidade científica internacional acredita haver associação causal entre a hiperhomocisteinemia e a aterogênese $e^{6-12}$. Esse desfecho patológico inicia-se com a ligação da homocisteína a proteínas diversas, determinando a homocisteinilação da albumina, da globulina, da hemoglobina e da lipoproteína de baixa densidade (LDL), entre outras ${ }^{1-3}$. Quanto maior a capacidade oxidativa do organismo, maior a homocisteinilação de proteínas e de moléculas de lipoproteína de baixa densidade (LDL). A homocisteinilação da LDL determina sua oxidação, etapa essencial da aterogênese, uma vez que a LDL oxidada é rapidamente fagocitada pelos macrófagos da parede arterial, induzindo a formação da célula espumosa e, numa etapa subseqüente, da placa ateromatosa ${ }^{7-8}$.

Sendo marcante a participação da hiperhomocisteinemia na aterogênese, torna-se fundamental que conheçamos seu metabolismo, seus fatores determinantes, sua importância clínica e suas formas de controle. Para isso, propusemonos a revisar a literatura, procurando consolidar os conhecimentos atuais acerca do metabolismo da homocisteína e de sua importância clínica na gênese de doenças cardiovasculares.

\section{MATERIAL E MÉTODO}

Este estudo consiste numa revisão bibliográfica de artigos científicos publicados na literatura nacional e internacional, selecionados por meio de pesquisa realizada eletronicamente no site do National Institute of Health (www.pubmed.org), utilizando-se, como descritores, "homocysteine" e/ou "hyperhomocysteine" e "cardiovascular disease" e/ou "cardiopathy". Foram selecionados artigos de revisão, metanálise, ensaios clínicos, estudos randomizados e guidelines que utilizaram seres humanos como população de estudo. Não foram utilizados limites de data, gênero ou idade. Das publicações listadas, 972 foram selecionadas por se aterem ao tema principal e, dessas, 57 foram utilizadas nesta revisão.

\section{O METABOLISMO DA HOMOCISTEÍNA}

A homocisteína (Hcy) é uma intermediária do metabolismo da metionina, possuindo um átomo de enxofre que a permite passar por uma série de reações bioquímicas comuns aos aminoácidos sulfurados. Sua forma molecular predominante no meio interno é a oxidada, encontrando-se ligada às proteínas ou à própria cisteína. É encontrada tanto no meio intracelular (quantidades mínimas) quanto no meio extracelular ${ }^{1-2}$. É sintetizada no organismo a partir dos aminoácidos metionina e cisteína, normalmente disponibilizados em quantidades satisfatórias pela dieta ocidental habitual. Suas principais fontes alimentares são as proteínas animais e as proteínas provenientes dos cereais ${ }^{3}$.

A principal função da Hcy é a manutenção de níveis séricos adequados de metionina, participando, dentro do 'ciclo metil', de três etapas metabólicas principais, detalhadas na Figura 1: a transmetilação, a remetilação e a transulfuração ${ }^{1-6}$. Na transmetilação, principal via de síntese da homocisteína, a metionina é sequencialmente convertida em homocisteína. $\mathrm{Na}$ remetilação, a metionina é regenerada a partir da Hcy, utilizando grupamentos metil fornecidos pelo N-metiltetra-hidrofolato (Metil-THF), na presença essencial de metilcobalamina, derivada da cianocobalamina (vitamina B12). Para isso, é necessário que o Metil-THF seja sintetizado no organismo, etapa bioquímica fundamental sob controle da enzima $\mathrm{N}^{5,10}$-metileno-tetrahidrofolato redutase (MTHFR), que tem sua ação controlada por feed-back negativo pela s-Adenosil-Metionina (AdoMet), enzima da etapa de transmetilação. Dessa forma, altos níveis séricos de metionina e, conseqüentemente, de AdoMet, previnem a formação de Metil-THF e, assim, reduzem a conversão da homocisteína em metionina. ${ }^{6}$ Finalmente, na transulfuração, disponível apenas no fígado, nos rins, no pâncreas e nos intestinos, a Hcy é hidrolisada a cisteína e $\alpha$-cetobutirato. Essa via metabólica é a principal responsável pelo catabolismo da homocisteína (degradando aproximadamente $50 \%$ da homocisteína circulante) e é dependente do piridoxal 5'-fosfato, gerado a partir da piridoxina (vitamina B6). 


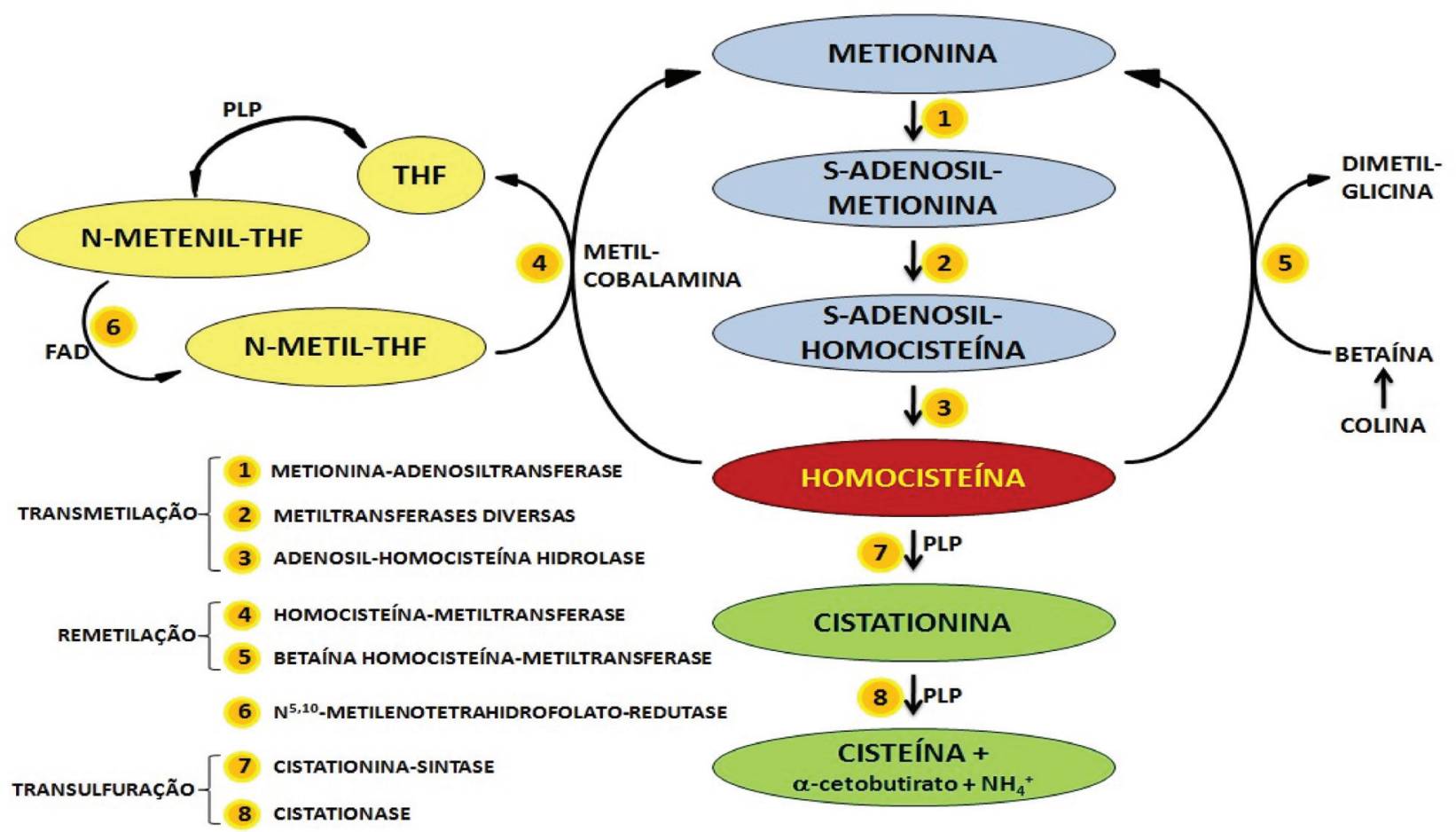

Figura 1. Etapas metabólicas da homocisteína (ciclo metil)

A Hcy participa, também, de outras reações bioquímicas. De maneira significativa, sofre conversão reversível à tiolactona, reação essa mediada principalmente pela metionil-tRNA-sintetase, existente em praticamente todas as células humanas, destacando-se as células endoteliais, os fibroblastos e as células tumorais mamárias. Essa reação é facilitada pela presença de altas concentrações séricas de homocisteína, sendo dificultada pela presença de concentrações adequadas de ácido fólico e vitamina B12. Uma vez formada, a tiolactona rapidamente reage com as proteínas, determinando sua homocisteinilação e, consequentemente, sua posterior degradação ${ }^{6}$. Outra reação habitual é sua ligação direta às proteínas, formando compostos tióis protéicos por meio da formação de radicais dissulfetos ${ }^{5}$

No meio intracelular, a transulfuração de Hcy em cistationina e/ou a remetilação à metionina mantém baixas suas concentrações ( 1 a $5 \mu \mathrm{mol} / \mathrm{L}$ ). A maior parte encontra-se na forma reduzida e livre, sendo o excesso exportado para o plasma. Já no meio extracelular, cerca de $70 \%$ da homocisteína encontrase ligada a proteínas (principalmente à albumina). $O$ restante circula como Hcy livre (forma reduzida) ou como dissulfetos mistos (Hcy-cisteína) $)^{1-3}$.

\section{PRINCIPAIS DETERMINANTES DE HIPERHOMOCISTEINEMIAS}

Utilizando valores acima do percentil 90, denominamos hiperhomocisteinemia (HHcy) quando os níveis séricos de Hcy em jejum estão acima de 14 a $16 \mu \mathrm{mol} / \mathrm{L}$ (média de $15 \mu \mathrm{mol} / \mathrm{L}$ ) ${ }^{1}$. As HHcy podem ser divididas em primárias (devidas à polimorfismos genéticos das enzimas envolvidas em seu metabolismo) e secundárias.

As mais graves HHcy são aquelas vistas na deficiência homozigótica da cistationina ß-sintase (Figura 1), herança autossômica recessiva e de baixa penetrância (1:335.000 indivíduos). Esses indivíduos apresentam homocisteinemias acima de $200 \mu \mathrm{mol} / \mathrm{L}$ e excretam diariamente mais de 10 $\mu \mathrm{mol} / \mathrm{L}$ de homocistina (dímeros de homocisteína), desenvolvendo uma síndrome clássica caracterizada por: homocistinúria, tromboses venosas prematuras, retardo mental e anormalidades esqueléticas. Já os indivíduos heterozigotos, condição bem mais prevalente $(0,3$ a $1,0 \%$ da população geral) apresentam níveis de Hcy apenas moderadamente elevados, sem tanta repercussão clínica. Mais comumente, uma mutação do gene que codifica a enzima $\mathrm{N}^{5,10}$-metilenotetrahidrofolato-redutase 
Lopes SLB, et al. A hiperhomocisteinemia como fator de risco cardiovascular.

(MTHFR), o polimorfismo C677T, determina uma forma branda de deficiência desta enzima, particularmente nos homozigotos TT, com manutenção de aproximadamente $50 \%$ de sua atividade biológica, o que incrementa significativamente os níveis séricos de Hcy. Nessa condição, por comprometimento do sítio de ligação da MTHFR com o ácido fólico, reduzse a remetilação da homocisteína, o que aumenta seus níveis séricos e incrementa o risco vascular destes pacientes. Na população geral observa-se uma prevalência de 1-5\% da homozigose TT e de 15 a $22 \%$ da heterozigose CT (distribuição variável nas populações estudadas) $)^{7-14}$. Outro polimorfismo da MTHFR que parece contribuir para aumento da Hcy sérica é o A1298C, existindo muita discordância na literatura acerca de seu real papel no desenvolvimento de patologias vasculares ${ }^{15-17}$.

Dentre as causas secundárias, principais determinantes de HHcy leves a moderadas, destacamse as deficiências das vitaminas participantes do ciclo metil (ácido fólico, cianocobalamina e piridoxina). A deficiência de ácido fólico determina um quadro de HHcy presumivelmente por dificuldades na remetilação da homocisteína, sendo encontrados níveis séricos até quatro vezes superiores àqueles mensurados em indivíduos com folatemias normais ${ }^{18-}$ 19. Já pacientes com deficiência de cianocobalamina apresentam, geralmente, elevações mais brandas da homocisteinemia, geralmente duas vezes superiores àquelas encontradas em indivíduos sem a deficiência vitamínica. Esse achado é tão significativo e reprodutivo que o encontro de HHcy tem sido utilizado, de forma sensível, como marcador da deficiência subclínica de vitamina B12 ${ }^{18,20-21}$, que deve, então, ser confirmada. A deficiência de piridoxina (vitamina B6) também tem sido responsabilizada, de forma não consensual, por incrementos dos níveis séricos de homocisteína. Apesar de essa relação ser bastante direta em estudos animais, em seres humanos parece não se comportar da mesma forma. O mecanismo de tal interferência em animais não está totalmente esclarecido, acreditando-se estar relacionado à redução da atividade da cistationina ß-sintase (Figura 1), dependente fundamentalmente do fosfato de piridoxal, derivado da piridoxina ${ }^{22-23}$.

Elevação leve a moderada dos níveis séricos de Hcy pode ser verificada, adicionalmente, nos pacientes com insuficiência renal crônica, que apresentam incremento dos níveis circulantes de Hcy em concordância com o nível de uremia apresentado. As razões desse aumento da Hcy sérica nesses indivíduos ainda não estão totalmente esclarecidas, acreditando dever-se mais à redução de sua metabolização pelo parênquima renal do que à redução de sua excreção urinária ${ }^{24}$. Outra condição clínica em que se verifica aumento dos níveis séricos de Hcy é o lúpus eritematoso sistêmico, cujos pacientes apresentam, em sua maioria, níveis mais baixos de folato e de cianocobalamina quando comparados à população em geral. Muito provavelmente são essas deficiências vitamínicas que determinam o incremento da Hcy sérica nesses pacientes ${ }^{25}$. Certas drogas utilizadas correntemente colaboram para aumentar a homocisteinemia, como a hidroclorotiazida, a carbamazepina, o ácido valpróico e a teofilina. Seus usuários beneficiam-se de sua titulação sérica, sendo imperativo normalizá-la quando fora da faixa aceitável ${ }^{26}$. Por fim, aumentos discretos, porém significativos, dos níveis plasmáticos de Hcy podem ser encontrados nos idosos e nos indivíduos tabagistas, hipercolesterolêmicos, hipertensos ou sedentários ${ }^{24-26}$.

\section{IMPORTÂNCIA CLIINICA DA HIPERHOMOCISTEINEMIA}

A HHcy, desde sua descrição inicial por McCully, em 196927, tem sido exaustivamente estudada quanto a sua participação na gênese de doenças vasculares. Estudando lesões arteriais de pacientes que faleceram com homocistinúria, McCully e Wilson ${ }^{28}$ demonstraram haver hiperplasia intimal, fibrose miointimal, e vacuolização e desnudação endotelial, com consequente trombose local comprometendo a luz arterial, já estreitada nesses indivíduos. Diante dessas alterações vasculares encontradas, os autores propuseram a hipótese de a hiperhomocisteinemia ser um fator etiológicometabólico de aterosclerose prematura ${ }^{28}$.

Entretanto, por não haver consenso a esse respeito, diversos autores se propuseram a verificar a relação da homocisteína sérica com o risco cardiovascular observado, estudos estes iniciados em 1976 por Wilcken e Wilcken ${ }^{29}$.

Em 1985, Murphy-Chutorian et al. ${ }^{30}$, analisando a homocisteinemia após carga de metionina em 138 homens cateterizados por suspeita de isquemia miocárdica, consideraram a Hcy como fator de risco para doença coronária, independentemente da idade, do hábito de fumar e da coexistência de hipertensão, diabetes mellitus ou hipercolesterolemia. Olszewski e Szostak ${ }^{31}$, em 1988 , estudaram 26 homens com infarto recente e observaram uma importante elevação da Hcy sérica nesses indivíduos quando comparados àqueles do grupo controle. Dois outros estudos reforçaram essa hipótese: no primeiro, um grupo de 170 homens com menos de 60 anos e com coronariopatia obstrutiva $>50 \%$ foi comparado a um grupo de indivíduos normais, evidenciando-se aumento 
significativo da Hcy sérica no primeiro grupo ${ }^{32}$. No segundo estudo, mais prospectivo, 14.916 médicos assintomáticos foram acompanhados por um período de cinco anos. Durante o acompanhamento, 271 indivíduos apresentaram infarto agudo do miocárdio e constituíram a população de estudo, em que se verificaram maiores níveis séricos de Hcy $(11,1+$ $4,0$ [SD] x 10,5 +2,8 $\mu \mathrm{mol} / \mathrm{mL} ; p=0,03)$. O risco relativo de infarto dos $5 \%$ com maiores níveis séricos, quando comparados com os $90 \%$ com menores níveis séricos, foi de 3,1 . Os autores concluíram que níveis séricos de Hcy moderadamente elevados estavam associados a risco subsequente de infarto do miocárdio, independentemente de outros fatores de risco coronariano ${ }^{33}$.

A década de 90 destacou-se pelo grande número de pesquisadores interessados nos polimorfismos genéticos da MTHFR. Em 1997, Anderson et al..$^{34}$ estudaram 200 pacientes submetidos a cineangiocoronariografia por infarto do miocárdio e analisaram o polimorfismo C677T da MTHFR. Quando comparados a 554 controles sadios, não foram significativas as prevalências da condição de homozigoto $(11,5 \%$ x 10,6\%) ou de heterozigoto (33,3\% x 32,1\%) entre os dois grupos, e concluíram que o polimorfismo não representava um fator de risco para coronariopatia ou infarto agudo do miocárdico em indivíduos bem nutridos. Posteriormente, em 1998, dois outros estudos chegaram à mesma conclusão, ambos realizados em países europeus ${ }^{35-36}$. Em 1999, Tsai et al. ${ }^{37}$ estudaram quatro polimorfismos genéticos de enzimas que participam do ciclo metil, a MTHFR (C677T), a cistationina ß-sintase (T133C e G919A) e a metionina sintase (A2756G), procurando correlacioná-los com a presença de coronariopatia prematura. Observaram que $8,5 \%$ dos pacientes coronariopatas (total de 376 casos) apresentavam HHcy, sendo que $47 \%$ desses não apresentavam deficiências de micronutrientes. Após análise dos dados, concluíram que os polimorfismos C677T da MTHFR e o T133C da cistationina ß-sintase implicavam maiores níveis séricos de Hcy e estavam associados a risco de aterosclerose coronariana prematura. Ainda em 1999, Wang et al. ${ }^{38}$ estudaram, também, os mesmos polimorfismos e concluíram que apenas os homozigotos TT carreavam maiores riscos de HHcy, correlacionando essa elevação sérica com maior potencial oxidativo medido pelo nível extracelular de superóxido dismutase nesses indivíduos. Friso et al. ${ }^{39}$, em 2002, avaliaram 470 indivíduos cuja anatomia coronariana tinha sido estudada (302 deles com coronariopatia), procurando estabelecer uma associação entre o polimorfismo genético e o nível sérico de ácido fólico. Concluíram que o polimorfismo A1298C da MTHFR não se associava a aumentos séricos significativos da Hcy, e que o risco coronariano era maior na subpopulação com desequilíbrio nutricional determinante de HHcy. Abu-Amero et al. ${ }^{40}$, em 2003, estudaram a distribuição dos genótipos C677T e A1298T da MTHFR em árabes, analisando uma amostra de 545 coronariopatas. Concluíram que a distribuição dessas duas variantes da MTHFR era semelhante e que nenhuma delas se correlacionava com coronariopatia na população árabe ${ }^{40}$. Nesse mesmo ano, Koch et al. ${ }^{41}$ investigaram a influência da HHcy e dos dois polimorfismos da MTHFR no risco de re-estenose em seis meses após angioplastia com "stent" em 800 pacientes coronariopatas sintomáticos. A taxa de re-estenose foi semelhante entre aqueles com HHcy e aqueles com homocisteinemia normal. Da mesma forma, mostrou-se semelhante nas duas mutações estudadas da MTHFR (C677T e A1298C). Em fevereiro de 2004, Toole et al. ${ }^{42}$ publicaram um bem conduzido estudo prospectivo objetivando determinar se altas doses (AD) de ácido fólico $(2,5 \mathrm{mg})$, cianocobalamina $(0,4 \mathrm{mg})$ e piridoxina (25 mg), ministradas no intuito de se reduzir a homocisteinemia total, eram capazes de reduzir o risco de acidente vascular cerebral recorrente (AVC) em um período de observação de 2 anos, quando comparadas com um regime de baixas dosagens (BD) destas mesmas vitaminas (20 $\mu \mathrm{g}$ de ácido fólico, $6 \mu \mathrm{g}$ de cianocobalamina e $200 \mu \mathrm{g}$ de piridoxina). Randomizaram um total de 3.680 adultos com AVC não debilitante em 56 hospitais universitários para receberem, obedecendo a um protocolo duplo-cego, um dos esquemas propostos de reposição vitamínica. Observou-se que o regime de altas doses reduziu a homocisteinemia sérica em $2 \mu \mathrm{mol} / \mathrm{L}$ a mais que 0 regime de baixas doses, sem reduzir, entretanto, o número de eventos primários (AVC) ou secundários (eventos coronarianos e óbitos) totais ( $A D=18,0 \%$ $x \mathrm{BD}=18,6 \%$ ). Não foram observadas diferenças significativas, também, quando se considerou isoladamente o risco de AVC recorrente $(A D=9,2 \% x$ $\mathrm{BD}=8,8 \%)$. Entretanto, demonstraram que a redução de $3 \mu \mathrm{mol} / \mathrm{L}$ da homocisteinemia implicava, nos 2 anos de seguimento, a redução do risco relativo de AVC $(B D=10 \% \times A D=12 \%)$, de eventos coronarianos $(B D=26 \%, A D=33 \%$ ) e de óbito ( $D B=16 \%, A D=$ $23 \%$ ), sendo que as diferenças encontradas entre os dois grupos não foram estatisticamente significativas. Adicionalmente, observaram uma associação positiva, persistente e cumulativa entre os níveis de homocisteinemia e a incidência dos eventos clínicos estudados ${ }^{42}$.

Mais recentemente, principalmente a partir de 2004, novos estudos têm dado importância à homocisteinilação protéica. Essa reação determina 
Lopes SLB, et al. A hiperhomocisteinemia como fator de risco cardiovascular.

alteração da função bioquímica e desarranjo da estrutura molecular das proteínas homocisteiniladas, com consequente resposta imune e formação de auto-anticorpos específicos. Altos níveis séricos de auto-anticorpos contra as proteínas homocisteiniladas (proteínas-Hcy), principalmente a albumina e a hemoglobina, têm sido demonstrados em algumas circunstâncias clínicas em que o substrato fisiopatológico é a agressão vascular, como o acidente vascular cerebral precoce, a coronariopatia aterosclerótica e o lúpus eritematoso sistêmico ${ }^{43-44}$. Undas et al. ${ }^{45}$, em junho de 2004 , demonstraram que pacientes masculinos com AVC apresentavam titulações de anticorpos anti-proteínas-Hcy pelo menos $50 \%$ maiores do que aquelas verificadas em indivíduos sadios, implicando a possibilidade desses antígenos aumentarem o risco de aterogênese. Os mesmos pesquisadores, em 2005, publicaram um estudo em que procuraram investigar a relação entre a presença de IgG anti-albumina-Hcy e a doença coronariana. Estudaram 88 homens com menos de 50 anos, sabidamente coronariopatas, e 100 indivíduos sadios. Observaram que a soropositividade para IgG anti-albumina-Hcy era 5 vezes mais frequente no grupo com coronariopatia que no grupo controle. Ademais, $41,2 \%$ dos pacientes com homocisteinemia $>14,5 \mu \mathrm{mol} / \mathrm{L}$ apresentavam IgG anti-albumina-Hcy, contra $25,5 \%$ dos pacientes com homocisteinemia abaixo desse valor. Observaram, também, uma fraca correlação entre a soropositividade para IgG anti-albumina-Hcy e os níveis séricos da Hcy, e nenhuma relação entre o polimorfismo C677T da MTHFR e a soropositividade para IgG antialbumina-Hcy. Concluíram, assim, que a presença de anticorpos anti-albumina-Hcy foi um preditor independente de coronariopatia em homens abaixo de 50 anos $^{46}$. Em 50 pacientes portadores de lúpus eritematoso sistêmico estudados por Padjas et al. ${ }^{25}$, observou-se que a prevalência de anticorpos anti-albumina-Hcy era maior que na população sadia $(50 \% \times 10 \%, P<0,001)$, com correlação positiva entre sua presença e os níveis séricos de Hcy e a duração da doença. O mesmo parece ocorrer em indivíduos renais crônicos submetidos a hemodiálise ${ }^{24}$. Posteriormente, verificou-se que mesmo indivíduos jovens sadios apresentavam níveis detectáveis desses auto-anticorpos, níveis esses que parecem aumentar progressivamente com o avançar da idade, promovendo um acúmulo de proteínas homocisteiniladas e cisteiniladas, provavelmente por redução da capacidade anti-oxidativa própria da fase de senescência ${ }^{47}$.

Evoluindo um pouco mais nessa mesma linha de raciocínio, alguns poucos grupos têm fomentado pesquisas laboratoriais objetivando verificar a relação entre a LDL, como consensual mediador de risco de aterosclerose, e os níveis séricos de Hcy. Demonstrou-se, particularmente em diabéticos, que a interação da Hcy-tiolactona com a LDL induz a formação de homocistamida-LDL (Hcy-LDL), sendo que essa reação aumenta a aterogenicidade da LDL. Essa hipótese foi testada por Ferreti et al. ${ }^{48}$, que incubaram lipoproteínas de indivíduos sadios e diabéticos com Hcy-tiolactona e observaram uma maior homocisteinilação das LDL nos diabéticos que nos indivíduos sadios. Posteriormente, incubaram células endoteliais de aorta humana com as LDL homocisteiniladas provenientes de indivíduos diabéticos e de indivíduos sadios, e demonstraram uma maior produção de peróxido nítrico pelas células expostas às LDL homocisteiniladas provenientes de indivíduos diabéticos, implicando menor capacidade anti-oxidativa e, consequentemente, maior grau de lesão endotelial nesses indivíduos. Muito semelhantemente, Dedoussis et al. ${ }^{49}$ analisaram as relações entre a distribuição dos genótipos do polimorfismo C677T da MTHFR, o nível de LDL oxidada e o consumo da dieta do tipo mediterrânea, rica em vitaminas e nutrientes antioxidantes. Estudaram 322 homens e 252 mulheres sem sinais clínicos de coronariopatia na região de Attica (Grécia). Em relação à genotipagem, não foram observadas diferenças entre os sexos; porém, os homozigotos TT apresentavam níveis de LDL oxidada $(70,8+26 \mathrm{mg} /$ $\mathrm{dL}$ ) estatisticamente superiores àqueles encontrados nos genótipos CT $(63,7+24 \mathrm{mg} / \mathrm{dL})$ ou CC $(51,0$ + $26 \mathrm{mg} / \mathrm{dL}$ ). Observaram, também, que a estrita adoção da dieta mediterrânea estava inversamente associada aos níveis de LDL oxidada, particularmente nos genótipos TT e CT. Esses dados permitiram aos autores concluir que a dieta mediterrânea poderia minimizar o risco coronariano em pessoas com potencial oxidativo aumentado.

Procurando equacionar dúvidas ainda existentes, alguns pesquisadores publicaram estudos visando a esclarecer os efeitos da reposição vitamínica no controle dos níveis de homocisteinemia e do risco de ateromatose prematura. Meleady et al. ${ }^{50}$, em 2003 , examinaram 1.458 pacientes em 11 países europeus, sendo 711 portadores de doenças vasculares. Observaram que o genótipo TT estava associado a níveis séricos mais elevados de homocisteína e mais baixos de folato, com um risco para doença vascular de 1,48 ('odds ratio'). Concluíram que o risco conferido pelo genótipo TT parece ser mediado pelo aumento da homocisteinemia na presença de baixos níveis séricos de folato. Klerk et al. ${ }^{51}$ concluíram uma meta-análise envolvendo 11.162 portadores de ateromatose coronariana e 12.758 controles. Observaram que o genótipo TT implicava 
maior tendência (16\%) de coronariopatia que o genótipo CC, e que a grande variação dos resultados obtidos pelos diversos trabalhos utilizados deveu-se particularmente ao nível sérico de folato. Concluíram que o distúrbio do metabolismo do folato, resultando em HHcy, apresentava relação causal com o aumento do risco coronariano. Porém, em 2005, publicou-se outra meta-análise em que os autores verificaram, após análise de 26.000 casos e 31.183 controles, que a associação do genótipo TT com doença coronariana deixava de ser significativa em países com alto consumo de folato, como os Estados Unidos, os países europeus e a Austrália. Para esses autores, a conclusão de estudos anteriores que correlacionavam o nível de folato com doença cardiovascular é duvidosa ${ }^{52}$. Em 2006, os investigadores do estudo multicêntrico Heart Outcomes Prevention Evaluation (HOPE 2) estudaram a reposição de ácido fólico (2,5 $\mathrm{mg}$ ), piridoxina (50 mg) e cianocobalamina (1 mg) em 5.522 pacientes com doença vascular já estabelecida. Após um período de acompanhamento de cinco anos, concluíram que os eventos primários (óbito por causas cardiovasculares, infarto do miocárdio e AVC) não foram significativamente reduzidos no grupo que recebeu a reposição vitamínica, com exceção dos acidentes vasculares cerebrais ${ }^{53}$. Em setembro de 2006, Undas et al. ${ }^{54}$ estudaram 12 pacientes com coronariopatia e 20 controles sadios, todos com homocisteinemia $>15 \mu \mathrm{mol} / \mathrm{L}$. Todos foram submetidos a reposição de ácido fólico ( $1 \mathrm{mg} / \mathrm{dia})$ por 3 ou 6 meses e tiveram titulados os anticorpos antialbumina-Hcy e anti-hemoglobina-Hcy. Observaram que a reposição de folato reduzia significativamente os níveis séricos de Hcy nos dois grupos de pacientes, e que os carreadores do alelo 677T da MTHFR apresentavam maiores titulações de anticorpos tanto antes como após a reposição de folato. Entretanto, as titulações de anticorpos somente eram reduzidas com a reposição de folato nos indivíduos sadios, não sendo observada essa redução nos pacientes coronariopatas. Concluíram, assim, que os indivíduos coronariopatas apresentavam resistência ao folato em reduzir os níveis de anticorpos anti-proteínas$\mathrm{Hcy}^{54}$. Em 2007, Ueland e Clarke ${ }^{55}$, em um editorial do periódico Clinical Chemistry, defenderam que a hiperhomocisteinemia era um fator de risco isolado para coronariopatias e um fator de risco pelo menos modesto para outras doenças cardíacas e para AVC, pelo menos em populações em que inexistia a adição obrigatória de ácido fólico aos alimentos. Reiteraram, por outro lado, o fato da reposição vitamínica não ser eficaz na redução do risco cardiovascular em indivíduos com doença já estabelecida e sob tratamento farmacológico adequado. Entretanto, não consideraram respondida a dúvida acerca da reposição vitamínica em longo prazo, sugerindo aguardar por novos estudos que esclarecessem melhor a questão ${ }^{55}$

Finalmente, poucos trabalhos foram publicados avaliando a população brasileira. Guerzoni et al. ${ }^{56}$, do Hospital de Base de São José do Rio Preto, estudaram o polimorfismo C677T da MTHFR em 127 indivíduos coronariopatas, não encontrando correlação entre os genótipos possíveis e o nível de Hcy sérica. Entretanto, os carreadores do genótipo TT apresentavam maior prevalência de coronariopatia severa (> 95\% de estenose) e de lesões biarteriais. Pereira et al. ${ }^{14}$, do Instituto do Coração da Universidade de São Paulo, utilizando a população recrutada para o estudo multicêntrico MASS II (558 pacientes com 2 ou 3 vasos comprometidos e fração de ejeção normal), observaram que o genótipo TT do polimorfismo C677T da MTHFR associava-se a maiores níveis de homocisteinemia e a maior mortalidade cardiovascular em 5 anos $(P=0,007)$. Finalmente, Rios et al. ${ }^{12}$, do Hospital Santa Izabel de Salvador, estudaram 772 indivíduos coronariopatas (480 caucasianos e 292 afro-descendentes), encontrando uma prevalência significativamente maior do alelo 677T em caucasianos que nos afrodescencentes $(28,1 \% \times 18,3 \%, p<0,001)$, culminando, nesses indivíduos homozigotos TT ou heterozigotos CT, num aumento do risco coronariano de 9,2 vezes (apenas nos indivíduos do sexo masculino) quando comparado aos indivíduos portadores do genótipo CC. Concluíram, assim, que a presença do alelo $677 \mathrm{~T}$ da MTHFR condicionava um aumento do risco coronariano em um padrão sexo-dependente em indivíduos brasileiros.

\section{DISCUSSÃO}

Apesar de ser um tópico de discussão relativamente recente, muito se conhece hoje sobre o metabolismo da homocisteína e sua frequente associação com a coronariopatia aterosclerótica, particularmente quando do aumento de seus níveis séricos. Inúmeros são os fatores determinantes da hiperhomocisteinemia, destacando-se os polimorfismos genéticos da MTHFR e a deficiência relativa das vitaminas essenciais ao ciclo metil. É certo que a hiperhomocisteinemia induz a formação de grupos tióis que se ligam a moléculas protéicas, determinando sua homocisteinilação, o que precipita o surgimento de auto-anticorpos dirigidos a essas proteínas homocisteiniladas. Já a homocisteinilação da molécula de LDL, processo acelerado em indivíduos com alto potencial oxidativo, parece acelerar a ateromatose vascular.

Parece prematuro concluir positivamente ou 
Lopes SLB, et al. A hiperhomocisteinemia como fator de risco cardiovascular.

negativamente acerca da necessidade de prevenção de vasculopatias ateroscleróticas nos portadores de HHcy. Entretanto, alguns subgrupos merecem consideração especial: indivíduos portadores de coronariopatia obstrutiva aparentemente não se beneficiam da redução dos níveis séricos elevados de homocisteína, não apresentando redução dos desfechos cardiovasculares primários (óbito) ou secundários (isquemia miocárdica traduzida por angina do peito ou infarto agudo do miocárdio). Já os indivíduos previamente acometidos por AVC isquêmicos se beneficiam desse controle, apresentando redução das taxas de recorrência de novos AVC. Também os hiperhomocisteinêmicos sem coronariopatia conhecida beneficiam-se do controle da hiperhomocisteinemia. Assim, é possível afirmar que todo indivíduo portador de algum fator de risco clássico para coronariopatia, principalmente aquele com história familiar de coronariopatia prematura, deve ter sua homocisteína sérica titulada, e aqueles com homocisteinemia acima de $15 \mu \mathrm{mol} / \mathrm{L}$ e com algum grau de resistência à sua correção com o controle da dieta e dos outros fatores de risco, devem realizar testes de genotipagem interessados no polimorfismo C677T da MTHFR. Parece sensato recomendar a esses indivíduos a utilização profilática diária de baixas doses de cobalamina (200 $800 \mu \mathrm{g} / \mathrm{dia}$ ) e de ácido fólico (400 a $800 \mu \mathrm{g} / \mathrm{dia}$ ), particularmente para os homozigotos TT, antes do desenvolvimento de coronariopatia sintomática. Essa recomendação também é válida para os portadores de hiperhomocisteinemia adquirida, cujos valores séricos persistem elevados, uma vez corrigidas as inadequações dietéticas habituais e os outros fatores de risco agregados. Cabe ressaltar que a adoção de uma dieta menos aterogênica e mais rica em grãos e cereais naturais (padrão da dieta mediterrânea) é, por si só, capaz de reduzir significativamente os níveis séricos de homocisteína, normalizando-os quando a elevação é discreta. Esse efeito positivo é observado, principalmente, quando a dieta usual é inadequada, rica em gorduras saturadas e proteínas animais.

Lopes SLB, Lopes HHMC, Vannucchi H. The hyperhomocysteinemia as a cardiovascular risk factor: actual perspectives. Rev Med (São Paulo). 2010 jan.-mar.;89(1):1-11.

ABSTRACT: Homocysteine, an intermediate of metionine metabolism, is considered, without a universal consent, an independent risk factor for ischemic cardiopathy. In order to review the literature, we conducted a bibliographical research interested in the association between hyperhomocysteinemia and cardiovascular risk. Conclusively, the hyperhomocysteinemia determines greater protein and LDL-cholesterol homocysteinilation, inducing specific autoantibodies against homocysteinilated proteins and higher circulating levels of oxidized LDLcholesterol, increasing the chances of vascular atherosclerosis. This association seems to depend, intrinsically, on the levels of two important co-factors involved in the methyl cycle, folic acid and cobalamin. Individuals with hyperhomocysteinemia, once supplemented with these vitamins, have reduced their cardiovascular risk and global mortality. However, this beneficial action seems to be restricted to people that use it as a form of primary prevention, since its use in individuals with atherosclerotic coronary artery disease did not reduce the incidence of ischemic events or mortality rates. It is concluded that the hiperhomocisteinemia is an independent cardiovascular risk factor, and should therefore be treated early, before the development of ischemic symptoms, with folic acid and cobalamin supplementation in order to control their serum levels and thus reduce the intrinsic cardiovascular risk. This seems to be important mainly in those individuals that, besides the hyperhomocysteinemia, carry others habitual risk factors for vascular atherosclerosis.

KEY WORDS: Homocysteine; Hyperhomocysteinemia; Atherosclerosis; Cardiovascular diseases; Risk factors.

\section{REFERÊNCIAS}

1. Stipanuk MH. Homocysteine, cysteine, and taurine. In: Shils ME, Olson JA, Shike M, Ross AC, editors. Modern nutrition in health and disease. 10th ed. Baltimore: Lippincott Willians \& Wilkins; 2006. p.54570.

2. Smith C, Marks AD, Lieberman M. Tetrahidrofolate,
Vitamine B12, and S-Adenosilmethionine. In: Smith C, Marks A, Liberman MA. Mark's basic medical biochemistry. 2nd ed. Baltimore: Lippincott Willians \& Wilkins; 2005. p.712-46.

3. Berg JM, Tymoczko JL, Stryer L. Biossíntese de aminoácidos. In: Berg JM, Tymoczko JL, Stryer 
L. Bioquímica. 5a ed. Rio de Janeiro: Guanabara Koogan; 2004. p.689-716.

4. Champe PC, Harvey RA, Ferrier DR. Aminoacid degradation and synthesis. In: Champe PC, Harvey RA. Lippincott's illustrated reviews: biochemistry. 4th ed. Baltimore: Lippincott Willians \& Wilkins; 2008. p.261-76.

5. Mayes PA. Structure and function of the water-soluble vitamins. In: Murray RK, et al. Harper's biochemistry. 25th ed. New York: McGraw-Hill; 2000. p.627-41.

6. Jakubowsky $\mathrm{H}$. Homocysteine thiolactone: metabolic origin and protein homocysteinylation in humans. $J$ Nutr. 2000;130:377S-381S.

7. Bydlowski SP, Magnanelli AC, Chamone DAF. Hiperhomocisteinemia e doenças vaso-oclusivas. Arq Bras Cardiol. 1998;71(1):69-76.

8. TrabettiE. Homocysteine, MTHFR gene polymorphisms, and cardio-cerebrovascular risk. J Appl Genet. 2008;49(3):267-82.

9. Alam MA, Husain SA, Narang R, Chauhan SS, Kabra M, Vasisht S. Association of polymorphism in the thermolabile 5, 10-methylene tetrahydrofolate reductase gene and hyperhomocysteinemia with coronary artery disease. Mol Cell Biochem. 2008;310(12):111-7.

10. Rassoul F, Richter V, Hentschel B, Geisel J, Herrmann W, Kuntze T. Plasma homocysteine levels \& 677C-->T methylenetetrahydrofolate reductase gene polymorphism in patients with coronary artery disease of different severity. Indian J Med Res. 2008;127(2):154-8.

11. Ilhan N, Kucuksu M, Kaman D, Ilhan N, Ozbay Y. The $677 \mathrm{C} / \mathrm{T}$ MTHFR polymorphism is associated with essential hypertension, coronary artery disease, and higher homocysteine levels. Arch Med Res. 2008;39(1):125-30.

12. Rios DL, D'Onofrio LO, Carvalho HG, Santos-Filho A, Galvão-Castro B. Sex-specific effect of the thermolabile C677T mutation in the methylenetetrahydrofolate reductase gene on angiographically assessed coronary artery disease in Brazilians. Hum Biol. 2007;79(4):453-61.

13. Bennouar N, Allami A, Azeddoug $H$, Bendris A, Laraqui A, El Jaffali A, et al. Thermolabile methylenetetrahydrofolate reductase C677T polymorphism and homocysteine are risk factors for coronary artery disease in Moroccan population. J Biomed Biotechnol. 2007;2007(1):ID80687. Available from: http://downloads.hindawi.com/journals/ jbb/2007/080687.pdf

14. Pereira AC, Miyakawa AA, Lopes NH, Soares PR, de Oliveira SA, Cesar LA, et al. Dynamic regulation of MTHFR mRNA expression and C677T genotype modulate mortality in coronary artery disease patients after revascularization. Thromb Res. 2007;121(1):25-32.

15. Freitas Al, Mendonça I, Guerra G, Brión M, Reis RP, Carracedo A, et al. Methylenetetrahydrofolate reductase gene, homocysteine and coronary artery disease: the $\mathrm{A} 1298 \mathrm{C}$ polymorphism does matter. Inferences from a case study (Madeira, Portugal). Thromb Res. 2008;122(2):648-56.

16. Sabbagh AS, Mahfoud Z, Taher A, Zaatari G, Daher $R$, Mahfouz RA. High prevalence of MTHFR gene A1298C polymorphism in Lebanon. Genet Test. 2008;12(1):75-80.

17. Ghazouani L. Homocysteine and methylenetetrahydrofolate reductase $\mathrm{C} 677 \mathrm{~T}$ and A1298C polymorphisms in Tunisian patients with severe coronary artery disease. J Thromb Thrombolysis. 2008;27(2):191-7.

18. Stabler SP, Marcell PD, Podell ER, Allen RH, Savage $D G$, Lindenbaum J. Elevation of total homocysteine in the serum of patients with cobalamin or folate deficiency detected by capillary gas chromatography-mass spectrometry. J Clin Invest. 1988;81(2):1466-74.

19. Kang SS, Wong PWK, Norusis M. Homocysteinemia due to folate deficiency. Metabolism. 1987;36(5):45862.

20. Lindenbaum J, Savage DG, Stabler SP, Allen RH. Diagnosis of cobalamin deficiency. II. Relative sensitivities of serum cobalamin, methylmalonic acid, and total homocysteine concentrations. Am J Hematol. 1990;34(2):99-107.

21. Savage DG, Lindenbaum J, Stabler SP, Allen RH. Sensitivity of serum methylmalonic acid and total homocysteine determinations for diagnosing cobalamin and folate deficiencies. Am J Med. 1994;96(3):23946.

22. Brattström $L$, Israelsson $B$, Norrving $B$, Bergqvist $D$, Thörne J, Hultberg B, et al. Impaired homocysteine metabolism in early-onset cerebral and peripheral occlusive arterial disease. Atherosclerosis. 1990;81(1):51-60.

23. Smolin LA, Bevenenga NJ. Accumulation of homocyst(e)ine in vitamin B6 deficiency: a model for the study of cystathionine-b-sinthase deficiency. J Nutr. 1982;112(7):1264-72.

24. Undas A, Kolarz M, Kopec G, Glowacki R, PlaczkiewiczJankowska E, Tracz W. Autoantibodies against $\mathrm{N}$-homocysteinylated proteins in patients on longterm haemodialysis. Nephron Dial Transplant. 2007;22(6):1685-9.

25. Padjas A, Undas A, Swadzba J, Musiał J. Antibodies to N-homocysteinylated albumin in patients with systemic lupus erythematosus. Pol Arch Med Wewn. 2007; 117(3):20-5.

26. Dierkes J, Luley C, Westphal S. Effect of lipid-lowering and anti-hypertensive drugs on plasma homocysteine 
Lopes SLB, et al. A hiperhomocisteinemia como fator de risco cardiovascular.

levels. Vasc Health Risk Manag. 2007;3(1):99-108.

27. Mccully KS. Vascular pathology of homocysteinemia: implications for the pathogenesis of arteriosclerosis. Am J Pathol. 1969;56(1):111-28.

28. Mccully KS, Wilson RB. Homocisteine therory of atherosclerosis. Atherosclerosis. 1975;22(2):215-27.

29. Wilcken DEL, Wilcken B. The pathogenesis of coronary artery disease. A possible role for methionine metabolism. J Clin Invest. 1976;57(4):1079-82.

30. Murphy-Chutorian DR, Wexman MD, Grieco AJ. Methionine intolerance: a possible risk factor for coronary artery disease. J Am Coll Cardiol. 1985;6(4):725-30.

31. Olszewski AJ, Szosta WB. Homocysteine content of plasma proteins in ischemic heart disease. Atherosclerosis. 1988;69(2-3):109-13.

32. Genest JJ Jr, McNamara JR, Salem DN, Wilson PW, Schaefer EJ, Malinow MR. Plasma homocyst(e)ine levels in men with premature coronary artery disease. J Am Coll Cardiol. 1990;16(5):1114-9.

33. Stampfer MJ, Malinow MR, Willett WC, Newcomer LM, Upson B, Ullmann D, et al. A prospective study of plasma homocyst(e)ine and risk of myocardial infarction in US physicians. JAMA. 1992;268(7):87781.

34. Anderson JL, King GJ, Thomson MJ, Todd M, Bair TL, Muhlestein JB, et al. A mutation in the methylenetetrahydrofolate reductase gene is not associated with increased risk for coronary artery disease or myocardial infarction. J Am Coll Cardiol. 1997;30(5):1206-11.

35. Girelli D, Friso S, Trabetti E, Olivieri O, Russo C, Pessotto R, Faccini G, et al. Methylenetetrahydrofolate reductase C677T mutation, plasma homocysteine, and folate in subjects from northern Italy with or without angiographically documented severe coronary atherosclerotic disease: evidence for an important genetic-environmental interaction. Blood. 1998;91(11):4158-63.

36. Brattström L, Wilcken DE, Ohrvik J, Brudin L. Common methylenetetrahydrofolate reductase gene mutation leads to hyperhomocysteinemia but not to vascular disease: the result of a meta-analysis. Circulation. 1998;98(23):2520-6.

37. Tsai MY. Genetic causes of mild hyperhomocysteinemia in patients with premature occlusive coronary artery diseases. Atherosclerosis. 1999;143(1):163-70.

38. Wang XL, Duarte N, Cai H, Adachi T, Sim AS, Cranney $G$, Wilcken DE. Relationship between total plasma homocysteine, polymorphisms of homocysteine metabolism related enzymes, risk factors and coronary artery disease in the Australian hospital-based population. Atherosclerosis. 1999;146(1):133-40.
39. Friso S, Girelli D, Trabetti E, Stranieri C, Olivieri O, Tinazzi E, et al. A1298C methylenetetrahydrofolate reductase mutation and coronary artery disease: relationships with $\mathrm{C} 677 \mathrm{~T}$ polymorphism and homocysteine/folate metabolism. Clin Exp Med. 2002;2(1):7-12.

40. Abu-Amero K, Wyngaard CA, Dzimiri N. Prevalence and role of methylenetetrahydrofolate reductase 677 C-->T and 1298 A-->C polymorphisms in coronary artery disease in Arabs. Arch Pathol Lab Med. 2003;127(1):1349-52.

41. Koch W, Ndrepepa G, Mehilli J, Braun S, Burghartz $M$, Lengnick $H$, et al. Homocysteine status and polymorphisms of methylenetetrahydrofolate reductase are not associated with restenosis after stenting in coronary arteries. Arterioscler Thromb Vasc Biol. 2003;23(12):2229-34.

42. Toole JF, Malinow MR, Chambless LE, Spence JD, Pettigrew LC, Howard VJ, et al. Lowering homocysteine in patients with ischemic stroke to prevent recurrent stroke, myocardial infarction, and death: the Vitamin Intervention for Stroke Prevention (VISP) randomized controlled trial. JAMA. 2004;291(5):565-75.

43. Jakubowski $H$, Zhang L, Bardeguez A, Aviv A. Homocysteine thiolactone and protein homocysteinylation in human endothelial cells: implications for atherosclerosis. Circ Res. 2000;87(1):45-51.

44. Jakubowski $\mathrm{H}$. New method for the determination of protein N-linked homocysteine. Anal Biochem. 2008;380(2):257-61.

45. Undas A, Perła J, Lacinski M, Trzeciak W, Kaźmierski R, Jakubowski H. Autoantibodies against $\mathrm{N}$-homocysteinylated proteins in humans - implications for atherosclerosis. Stroke. 2004;35(6):1299-304.

46. Undas $A$, Jankowski $M$, Twardowska M, Padjas A, Jakubowski H, Szczeklik A. Antibodies to $\mathrm{N}$-homocysteinylated albumin as a marker for early-onset coronary artery disease in men. Thromb Haemost. 2005;93(2):346-50.

47. Rossi R, Giustarini D, Milzani A, Dalle-Donne I. Cysteinylation and homocysteinylation of plasma protein thiols during ageing of healthy humans. J Cell Mol Med. 2008;13(9B):3131-40.

48. Ferretti G, Bacchetti T, Rabini RA, Vignini A, Nanetti $\mathrm{L}$, Moroni $\mathrm{C}$, et al. Homocysteinylation of low-density lipoproteins (LDL) from subjects with Type 1 diabetes: effect on oxidative damage of human endothelial cells. Diabet Med. 2006;23(4):808-13.

49. Dedoussis GV, Panagiotakos DB, Chrysohoou C, Pitsavos C, Zampelas A, Choumerianou D, et al. Effect of interaction between adherence to a Mediterranean diet and the methylenetetrahydrofolate reductase 677C-->T mutation on homocysteine concentrations 
in healthy adults: the ATTICA Study. Am J Clin Nutr. 2004;80(4):849-54.

50. Meleady R, Ueland PM, Blom H, Whitehead AS, Refsum H, Daly LE, Vollset SE, et al. Thermolabile methylenetetrahydrofolate reductase, homocysteine, and cardiovascular disease risk: the European Concerted Action Project. Am J Clin Nutr. 2003;77(1):63-70.

51. Klerk M, Verhoef $P$, Clarke R, Blom HJ, Kok FJ, Schouten EG. MTHFR 677C-->T polymorphism and risk of coronary heart disease: a meta-analysis. JAMA. 2002;288(16):2023-31.

52. Lewis SJ, Ebrahim S, Davey SG. Meta-analysis of MTHFR 677C->T polymorphism and coronary heart disease: does totality of evidence support causal role for homocysteine and preventive potential of folate? BMJ. 2005;331(7524):1053-9.
53. Lonn E, Yusuf S, Arnold MJ, Sheridan P, Pogue J, Micks $M$, et al. Homocysteine lowering with folic acid and $\mathrm{B}$ vitamins in vascular disease. $\mathrm{N}$ Engl $\mathrm{J}$ Med. 2006;354(15):1567-77.

54. Undas A, Stepień E, Glowacki R, Tisończyk J, Tracz W, Jakubowski H. Folic acid administration and antibodies against homocysteinylated proteins in subjects with hyperhomocysteinemia. Thromb Haemost. 2006;96(3):342-7.

55. Ueland PM, Clark, R. Homocysteine and cardiovascular risk: considering the evidence in the context of study design, folate fortification, and statistical power. Clin Chem. 2007;53(5):807-9.

56. Guerzoni AR, Pavarino-Bertelli EC, Godoy MF, Graça CR, Biselli PM, Souza DR, et al. Methylenetetrahydrofolate reductase gene polymorphism and its association with coronary artery disease. São Paulo Med J. 2007;125(1):4-8. 